SLAC-PUB-8079

March 1999

\title{
SIMULATION OF EXPERIMENTAL BACKGROUND USING FLUKA
}

\author{
R. Mitchell et al.
}

\author{
Stanford Linear Accelerator Center \\ Stanford University \\ Stanford, CA 94309 USA
}

Presented at the 4th Workshop on Simulating Accelerator Radiation Environments, September 14-16, 1998, Knoxville, Tennessee

Work supported by Department of Energy contracts DE-AC03-76SF00515 and DOE DE-FG05-91ER40627. 


\title{
SIMULATION OF EXPERIMENTAL BACKGROUND USING FLUKA
}

\author{
R. Mitchell ${ }^{\ddagger}$, A. Fasso ${ }^{\dagger}$, S. Rokni ${ }^{\dagger}$ \\ ${ }^{\dagger}$ Stanford Linear Accelerator Center, P.O. Box 4349, Stanford, CA 94039 \\ ${ }^{*}$ Department of Physics, University of Tennessee, Knoxville, TN 37916
}

\begin{abstract}
In November 1997, Experiment T423 began acquiring data with the intentions of understanding the energy spectra of high-energy neutrons generated in the interaction of electrons with lead. The following describes a series of FLUKA simulations studying (1) particle yields in the absence of all background; (2) the background caused from scattering in the room; (3) the effects of the thick lead shielding which surrounded the detector; (4) the sources of neutron background created in this lead shielding; and (5) the ratio of the total background to the ideal yield. In each case, particular attention is paid to the neutron yield.
\end{abstract}

\section{T423: Measurement of Neutron Energy Spectra using TOF}

Experiment T423 was conducted in the FFTB (Final Focus Test Beam) facility at SLAC. Neutrons were produced when the $20 \mathrm{GeV}$ electron beam collided with a $2 \mathrm{~cm}$ thick lead target. The TOF distance was approximately 6 meters. Neutrons ranging in energies from 2 to $200 \mathrm{MeV}$ were detected in two detectors. A 5" liquid scintillator was located at 26 degrees and a 2" plastic scintillator was used at 38 degrees. The following simulations only consider the yield at the 5" detector and do not incorporate detector efficiencies.

\section{Background Studies}

A series of FLUKA simulations were performed aiming to answer the following five questions:

(i) Which particles and what particle yields are found at the detector position in the absence of background and what are the shapes of their energy spectra? This will subsequently be referred to as the "perfect signal".

(ii) How much background does the room cause? What is the relative contribution made by each room component?

(iii) What are the effects of the lead shielding surrounding the detector? How effective is this in reducing the background?

(iv) Which particles cause neutrons in the lead shielding?

(v) How does the total background compare with the perfect signal?
The Monte Carlo code FLUKA was used in attempting to answer these questions because of its ability to treat photonuclear interactions with a wide range of energies [1].

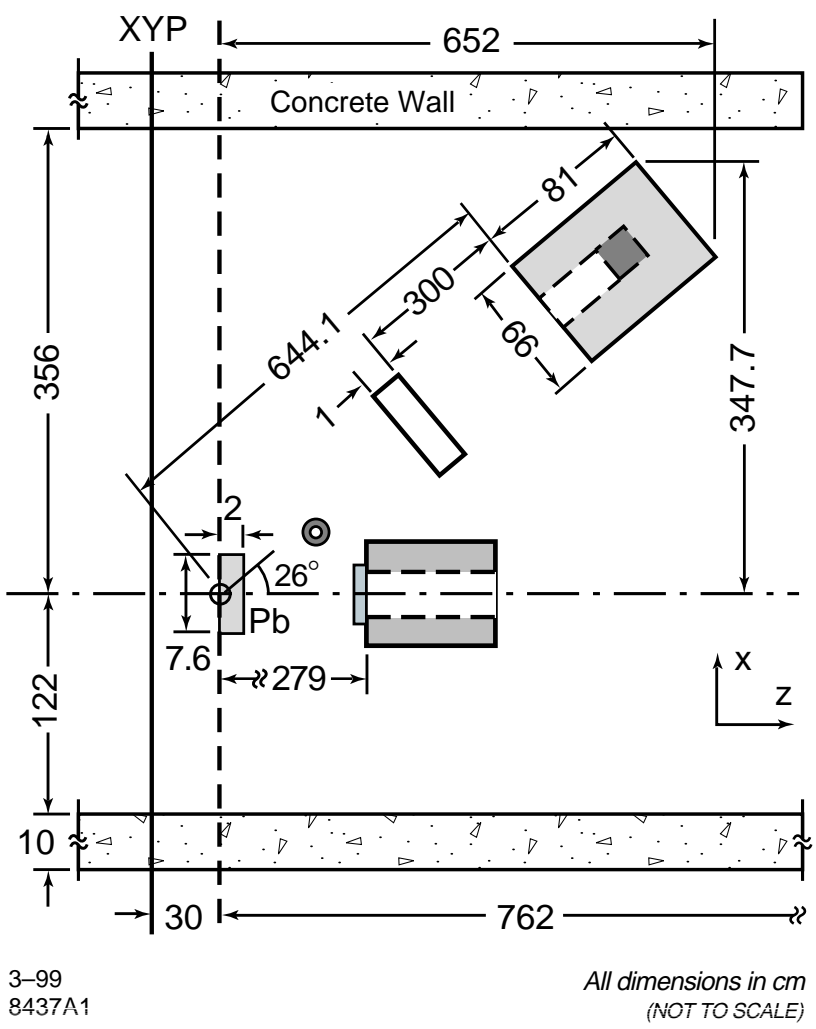

Figure 1. The components of the experiment that were included in the simulations. 
Eight features of the experimental area were taken into consideration in various combinations by the background simulations (figures 1, 2 and 3). (1) The target was a $7.62 \mathrm{~cm} \times 7.62 \mathrm{~cm} \times 2 \mathrm{~cm}$ block of lead with the $20 \mathrm{GeV}$ electron beam normal to and centered on its $7.62 \mathrm{~cm} \times 7.62 \mathrm{~cm}$ face. (2) The detector package was a $66 \mathrm{~cm} \times 81 \mathrm{~cm} \times 30 \mathrm{~cm}$ box of lead shielding which housed the detector. A rectangular opening faced the target and lead to the detector (figure 2). (3) The detector was an $11.26 \mathrm{~cm}$
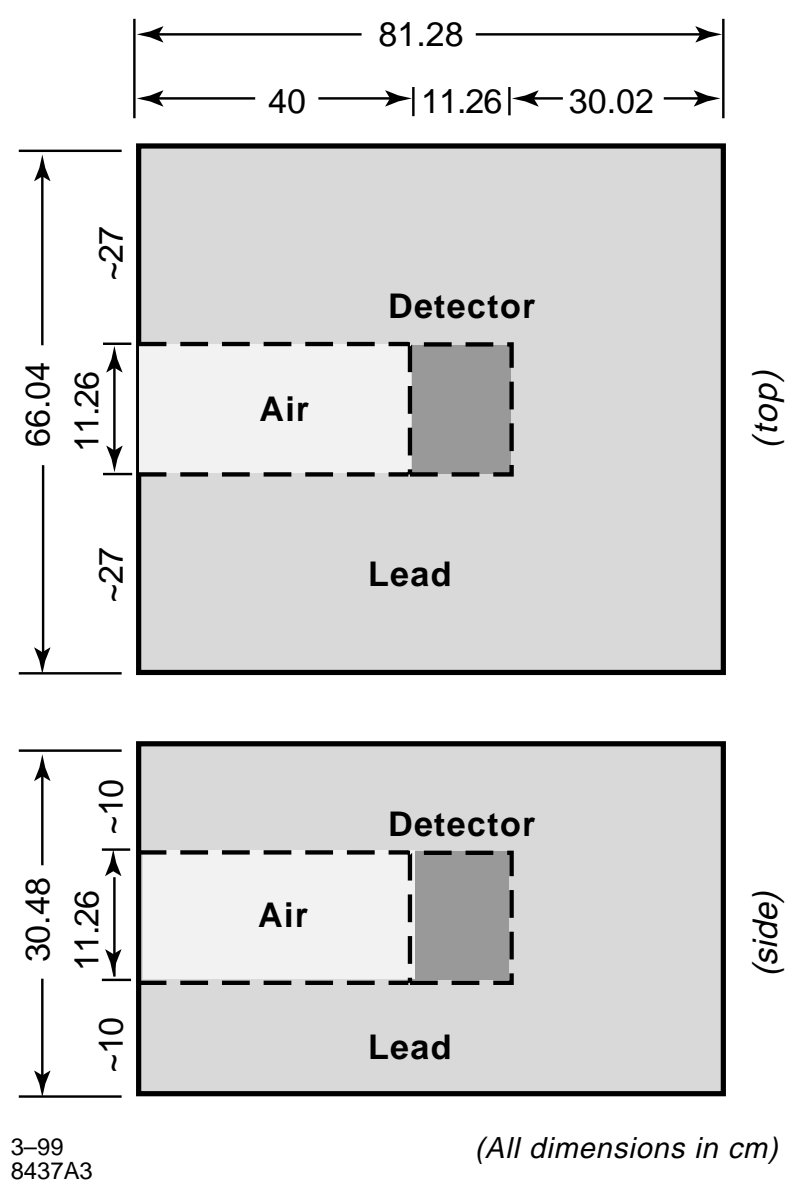

Figure 2. A model of the detector and the lead shielding surrounding it.

$\times 11.26 \mathrm{~cm} \times 11.26 \mathrm{~cm}$ square box which scored and then killed particles as they entered. (4) A shadow shield was occasionally used to block the detector from particles travelling directly from the target. In some simulations a large shadow shield $(61 \mathrm{~cm} \times 1 \mathrm{~cm}$ $\times 25 \mathrm{~cm}$ ) was used to block the entire front face of the detector package (the detector plus the lead shielding), while in others a small shadow shield $(25 \mathrm{~cm} \times 1 \mathrm{~cm} \times 20 \mathrm{~cm})$ was used to block only the front face of the detector. In either case, it was located nearly halfway $(343 \mathrm{~cm}$ from the target) between the target and the detector and killed all of
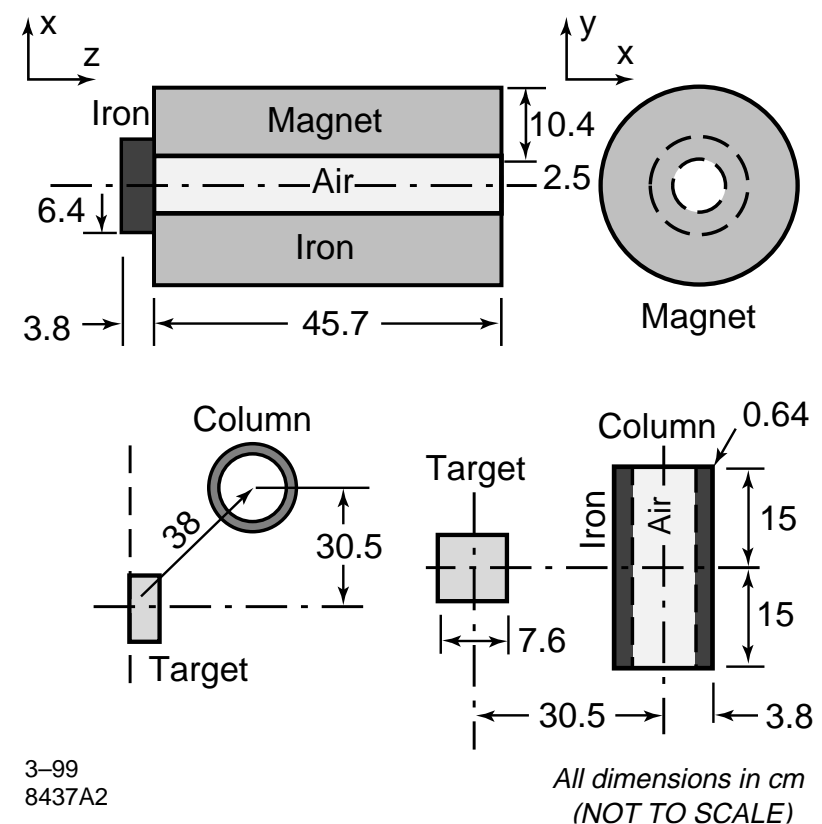

3-99

8437A2

(NOT TO SCALE)

Figure 3. Models of the downstream quadrupole magnet and the iron column.

the particles which came in contact with it. (5) The concrete walls (including the floor and ceiling) were all assumed to be $10 \mathrm{~cm}$ thick. They were made to extend from $30 \mathrm{~cm}$ upstream of the target to approximately $100 \mathrm{~cm}$ downstream of the far end of the detector package. The room was $287 \mathrm{~cm}$ high and $478 \mathrm{~cm}$ wide. (6) The downstream quadrupole magnet was assumed to be a hollow cylinder of iron $46 \mathrm{~cm}$ long with an inner radius (filled with vacuum) of $2.5 \mathrm{~cm}$ and an outer radius of $13 \mathrm{~cm}$. A cylindrical iron cap $3.8 \mathrm{~cm}$ long and with a radius of $5.7 \mathrm{~cm}$ was placed in front of the magnet's hole. (7) A hollow iron column was included $38 \mathrm{~cm}$ from the target. (8) Air filled in the rest of the room.

Five main simulations were performed. Throughout this paper, the five simulations will be referred to as the following:

SPHERES (figure 4) is the simulation which ignores all background (the "perfect signal"). Here, the only components of the room taken into account are the target and the air. Because of the azimuthal symmetry in this simple simulation, particle yield was scored on the surface of a sphere. The original results of FLUKA (scoring using USRYLD) were given as particles/GeV/sr/e. This was converted to particles $/ \mathrm{GeV} / \mathrm{cm}^{2} / \mathrm{e}$ and then normalized to the area of the front face of the detector to give (particles entering detector)/GeV/e. All of the results which follow are normalized to the size of the detector, i.e. 
measured in the units (particles entering detector)/GeV/e, and are thus directly comparable.

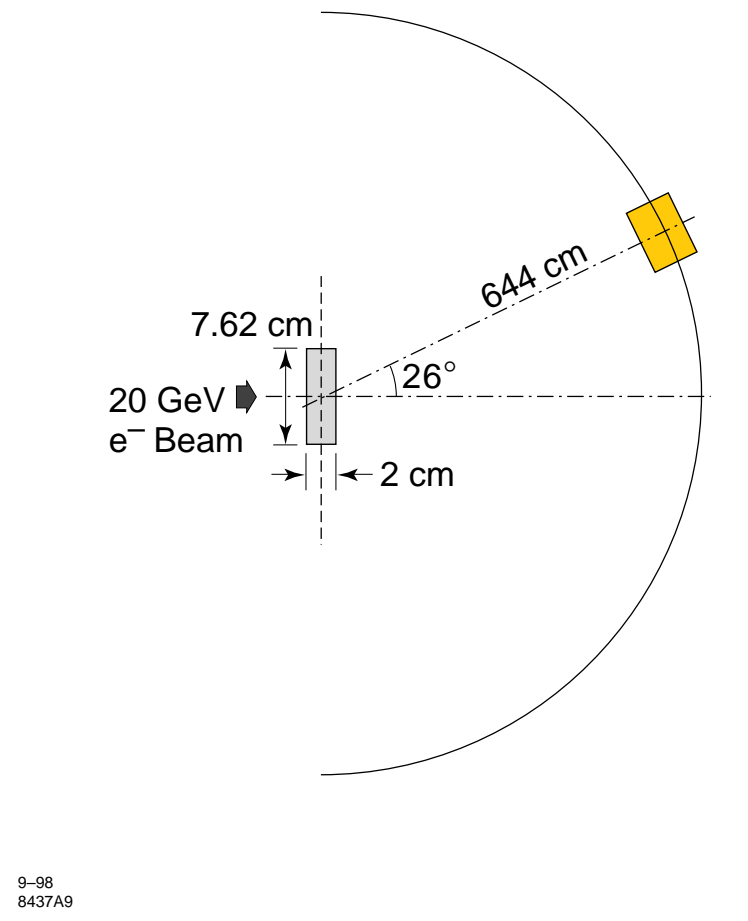

Figure 4. The geometry used in the SPHERES simulation.
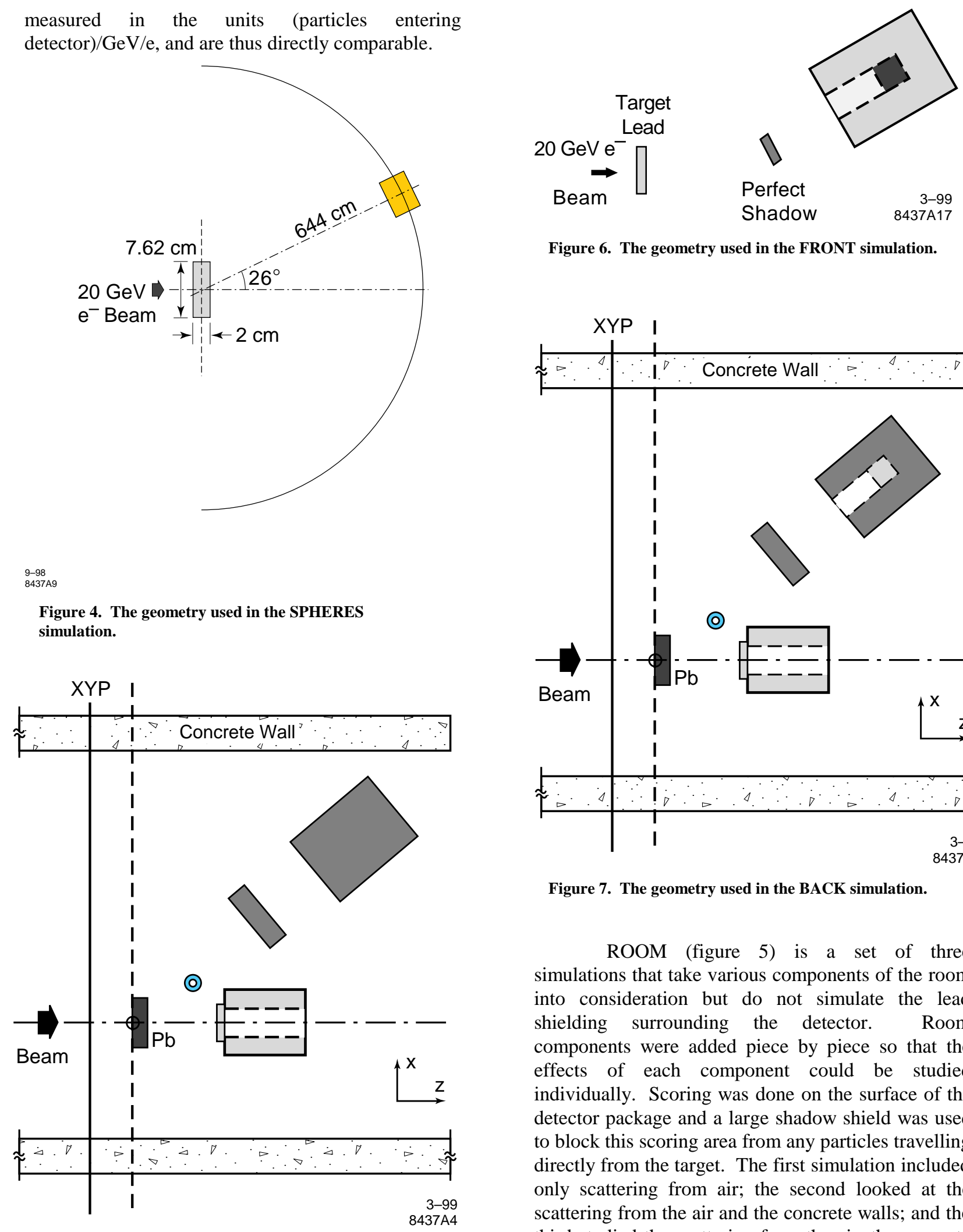

Figure 6. The geometry used in the FRONT simulation.

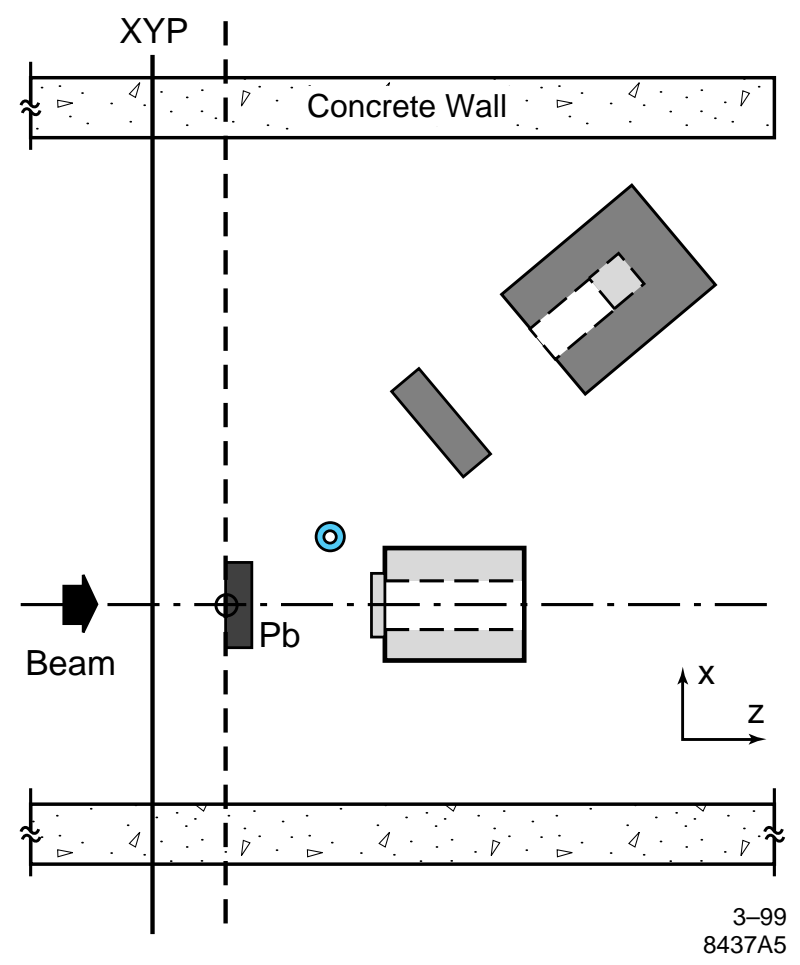

Figure 7. The geometry used in the BACK simulation.

ROOM (figure 5) is a set of three simulations that take various components of the room into consideration but do not simulate the lead shielding surrounding the detector. Room components were added piece by piece so that the effects of each component could be studied individually. Scoring was done on the surface of the detector package and a large shadow shield was used to block this scoring area from any particles travelling directly from the target. The first simulation included only scattering from air; the second looked at the scattering from the air and the concrete walls; and the third studied the scattering from the air, the concrete walls, the downstream magnet, and the iron column. 
FRONT (figure 6) is a simulation that only considers the background caused by the front surface of the detector package consisting of the lead shielding. A small shadow shield was used that was large enough to block the detector but small enough to allow particles to travel directly from the target to the lead surrounding it. The room was not included.

BACK (figure 7) uses a large shadow shield to cover the entire front surface of the lead shielding. All of the room components are simulated. Thus, this simulation describes the scattering from the room making it through the lead shielding.

TOTAL (figure 8 ) is a complete simulation of the background. It simulates all of the room components and uses a small shadow shield to allow particles travelling directly from the target to interact with the front surface of the lead shielding. Notice that this should correspond with the addition of FRONT (scattering from the front surface of the lead shielding) and BACK (scattering from the room).

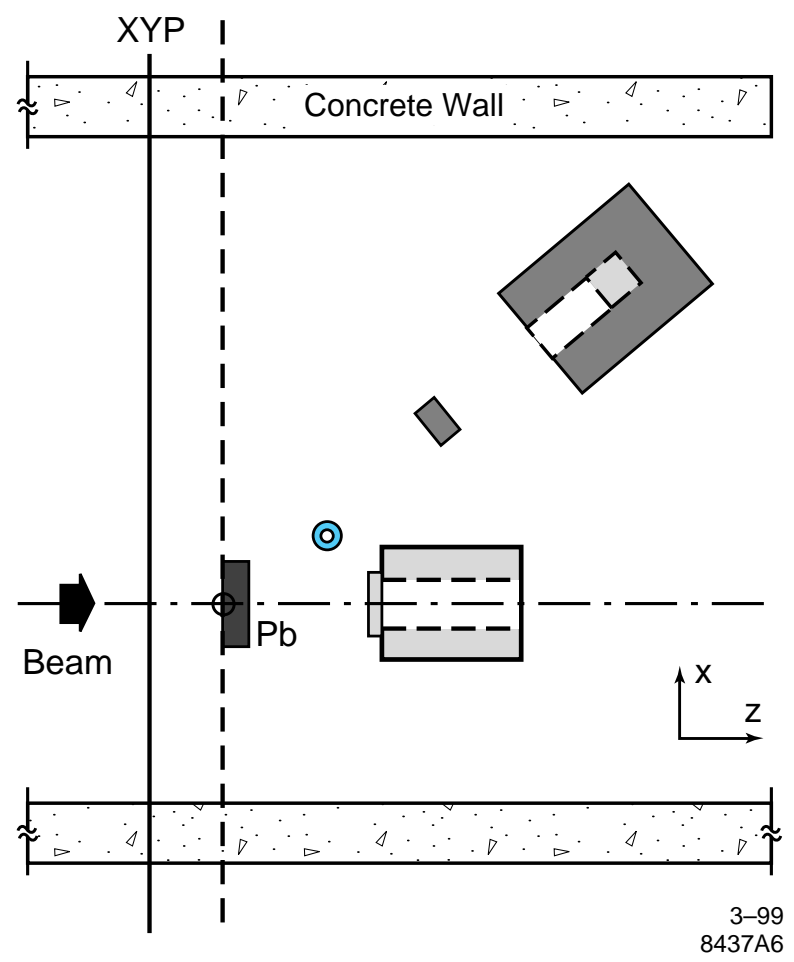

Figure 8. The geometry used in the TOTAL simulation.

In order to increase statistics, FRONT, BACK, and TOTAL were run in two steps. First, photons, neutrons, and pions that reached the outside surface of the detector package (the lead surrounding the detector) were scored and their energies, positions, trajectories, and statistical weights were written to files. Typically, these files consisted of 2000 to 4000 particles. Then, using these files as source files, particles were sent through the lead shielding and the yields were scored at the detector. A normalization scheme was worked out to provide the correct weighting [2].

Two forms of biasing were consistently used throughout the following simulations -- leading particle biasing was used in all electromagnetic interactions under $10 \mathrm{GeV}$, and the inelastic decay length of photons was reduced by a factor of 0.02 . When measuring the electron/positron, photon, and neutron yields, the cutoff kinetic energy for electrons and photons was $1 \mathrm{MeV}$. When measuring the pion and muon yields, the cutoff energy was $130 \mathrm{MeV}$.

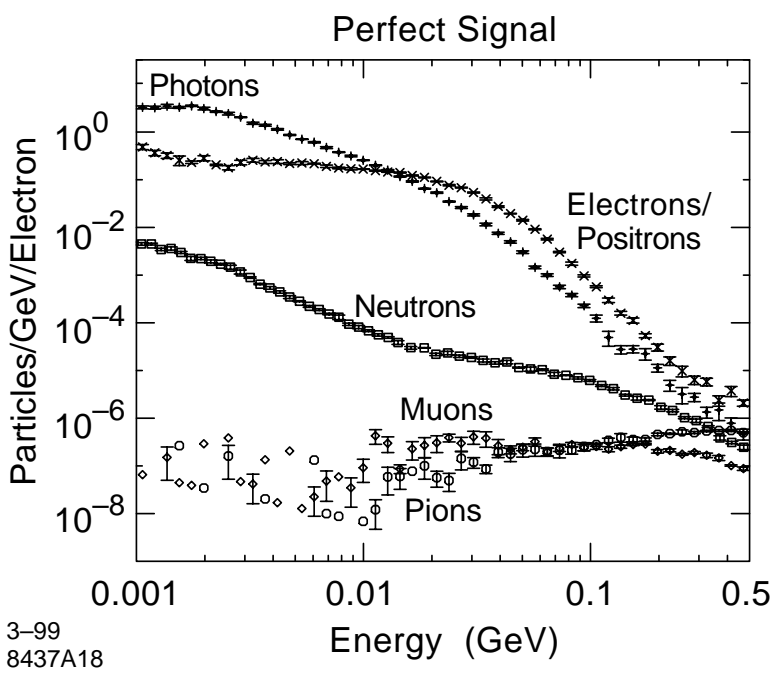

Figure 9. Particle yields in the absence of all background (from SPHERES).

\section{The "Perfect Signal"}

The particle yields in the absence of background were found using the SPHERES simulation (figure 9). Electrons/positrons, photons, neutrons, charged pions, and muons are all observed to reach the detector position. The kaon yield was negligible, and was also discarded in the remaining simulations. Notice that the neutron yield drops by approximately two orders of magnitude from $2 \mathrm{MeV}$ to $100 \mathrm{MeV}$. The particle yields recorded in figure 9 provide the standard by which the magnitude of the background may be judged.

\section{The Room Background}

Studies of the room background were performed using the ROOM simulations. It should be remembered that these results describe what the yield 
would have looked like had the detector not been heavily shielded with lead. Further, it is important to note that the background has been isolated by including an ideal shadow shield (a black hole) blocking the detector from direct hits. Figure 10 shows the particle yields at the detector position when the entire room is included (concrete walls, downstream magnet, and iron column).

Room Background (Concrete, Magnet, Column) - No Shielding at Detector

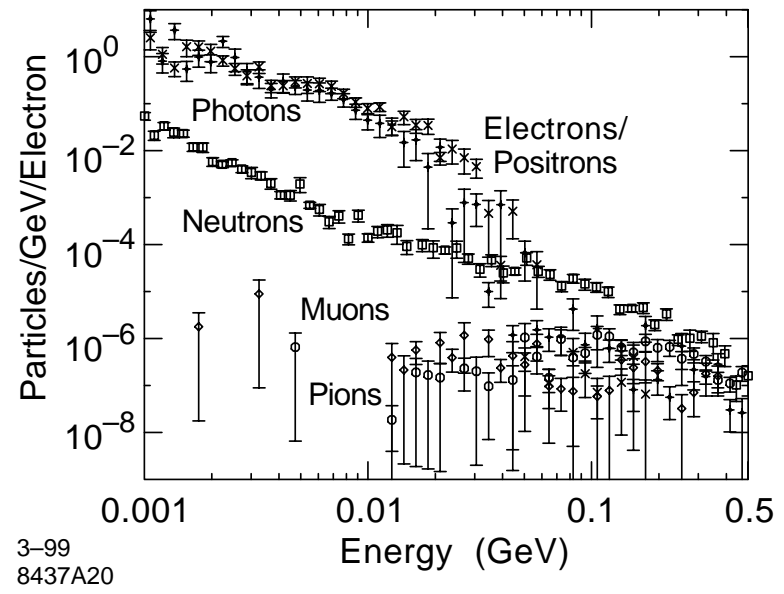

Figure 10. Particle yields at the detector position when the room is included (from ROOM).

\section{Contributions to the Photon Background}

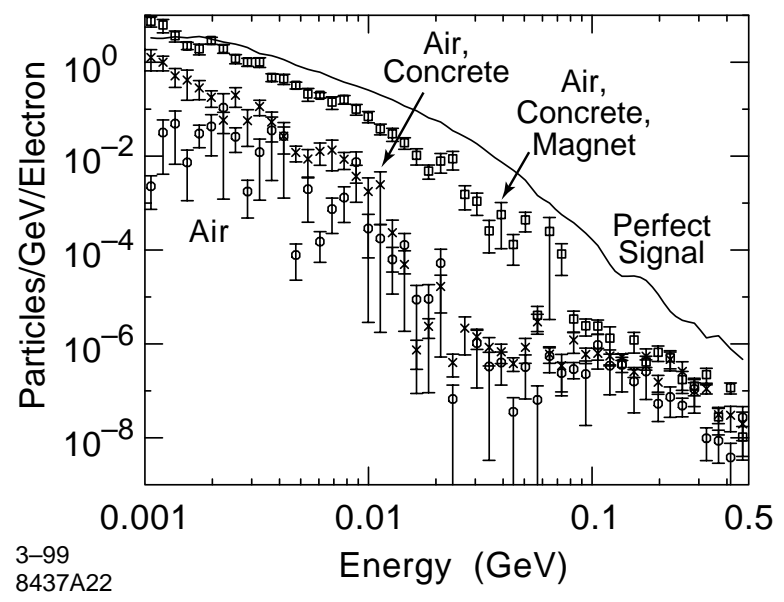

Figure 11. The effects of various room components on the photon background (from ROOM).

In addition to simulating the entire room background, simulations were performed which investigate the contributions of each room component. Figure 11 shows the effects of room components on the photon background. The smallest yield corresponds to the simulation that used only air. Everything here has scattered around the shadow shield. Next, one finds a small increase when the concrete walls are added to the room. Finally, there is nearly an order of magnitude increase when the magnet is included. The solid line corresponds to the photon yield given by the SPHERES simulation. From figure 11, one may conclude that the downstream magnet plays the most important role in the photon room background.

The same analysis described above for photons was performed also for neutrons. The results are shown in figure 12. Here, it is evident that both the inclusions of the magnet and of the concrete walls substantially effect the neutron room background.

\section{Contributions to the Neutron Background}

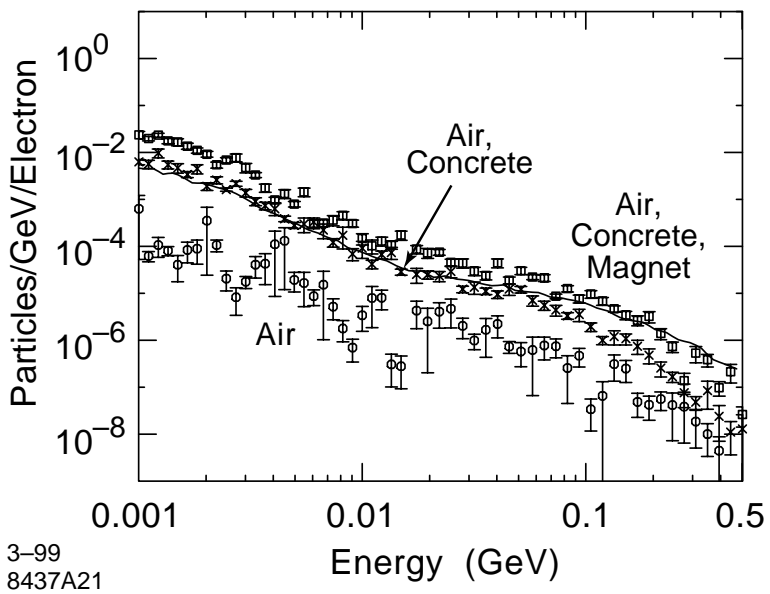

Figure 12. The effects of various room components on the neutron background (from ROOM).

\section{The Lead Shielding}

The shielding which surrounds the detector has two distinct effects (figure 13). First, there will be some amount of augmentation given to the perfect signal due to the scattering from the front surface of the lead. Second, the lead will reduce the amount of room background making it to the detector. These two effects may be studied separately using the FRONT and BACK simulations, respectively.

The signal augmentation may be studied by comparing the results of the FRONT simulation with the perfect signal (figure 14). The addition of the augmentation to the perfect signal would give the total yield in the detector if there were no scattering from the room. Figure 14 shows that the ratio of perfect signal to augmentation increases as the energy increases, beginning at around a factor of two and ending at nearly an order of magnitude. 
The positive effect of the lead shielding is in reducing the room background. Figure 15 compares the ROOM simulation (the room background with no lead shielding) with the BACK simulation (the room background with lead shielding). On average, it seems that the lead decreases the neutron background by a factor of two.

(a) Signal Augmentation

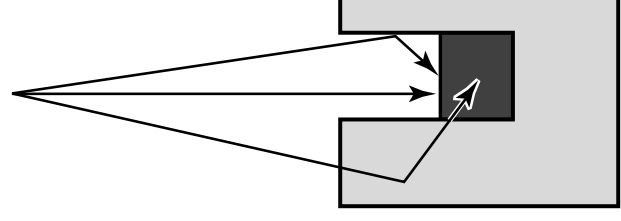

(b) Background Reduction $3-99$ 8437A8

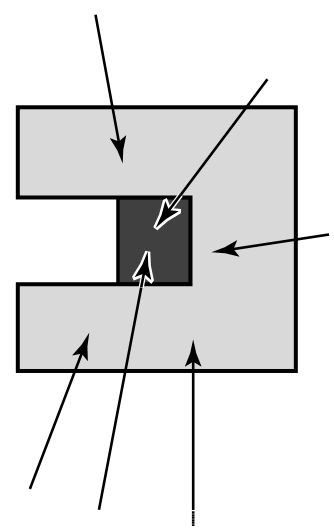

Figure 13. Two distinct effects of the shielding which surrounds the detector.

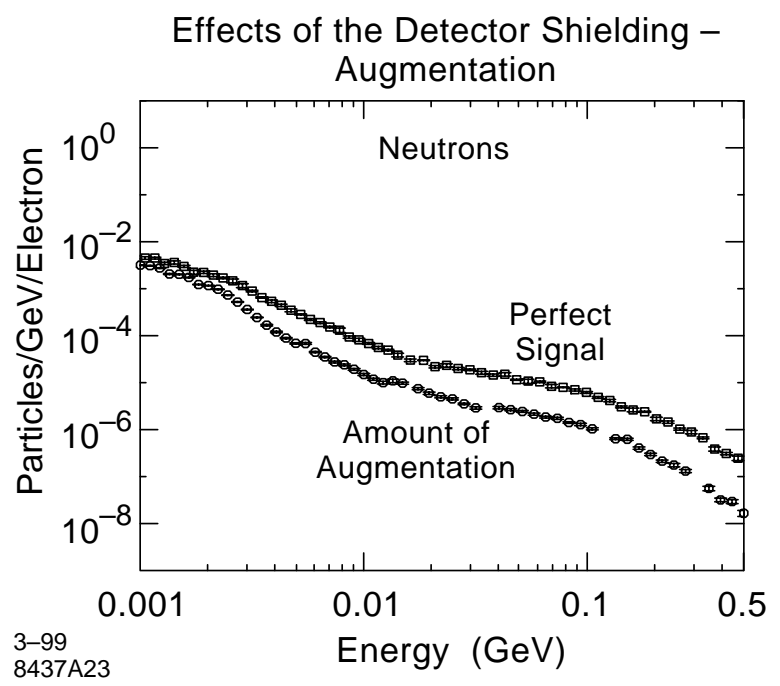

Figure 14. The signal augmentation -- the enhancement of the signal due to the background from the front of the lead shielding (from FRONT and SPHERES).

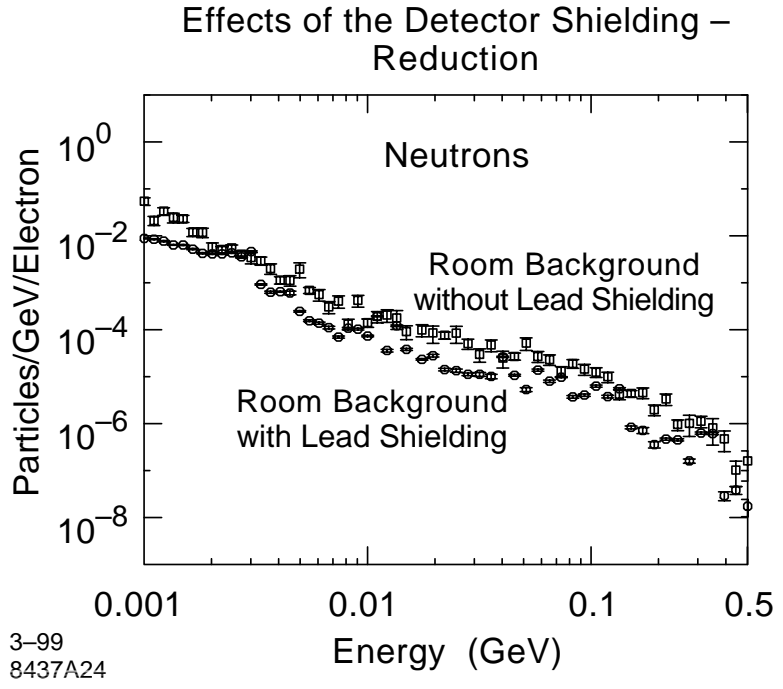

Figure 15. The background reduction (from BACK and ROOM).

\section{Sources of the Neutron Background}

The sources of the neutron background created in the lead may be studied with the TOTAL simulation. Using the two-step process described above, the effects of the photons, neutrons, and pions that reach the detector package could be studied individually. For instance, the effect of the photons interacting with the lead shielding could be studied by sampling from the file containing a list of all the photons that reached the lead shielding in the first step. All of the neutrons created from a simulation performed in this manner must originate from photonuclear interactions. In the same manner, the yield of neutrons originating from pions or other neutrons could be studied. Figure 16 shows the results of this study. The results show that neutron production in the lead is dominated by $(\mathrm{n}, \mathrm{xn})$ reactions and that the shape of this spectrum mirrors that of the signal (figure 9). The pions create approximately an order of magnitude less neutrons in the lead than the neutrons do. Again, the shape of the spectrum corresponds with that of the signal. The photons reacting in the lead appear to be unimportant, especially above $10 \mathrm{MeV}$ where they virtually disappear.

\section{Comparison of the Total Background with the Perfect Signal}

The comparison of the results from the SPHERES simulation with the TOTAL simulation allows a determination of the ratio of the perfect signal to the total background. Figure 17 indicates that this ratio 
is, within error, 1:1. This implies the ratio of the measured signal (the total background plus the perfect signal) to the total background is approximately $2: 1$.

\section{Background Neutrons Created by Photons, Pions, and Neutrons}

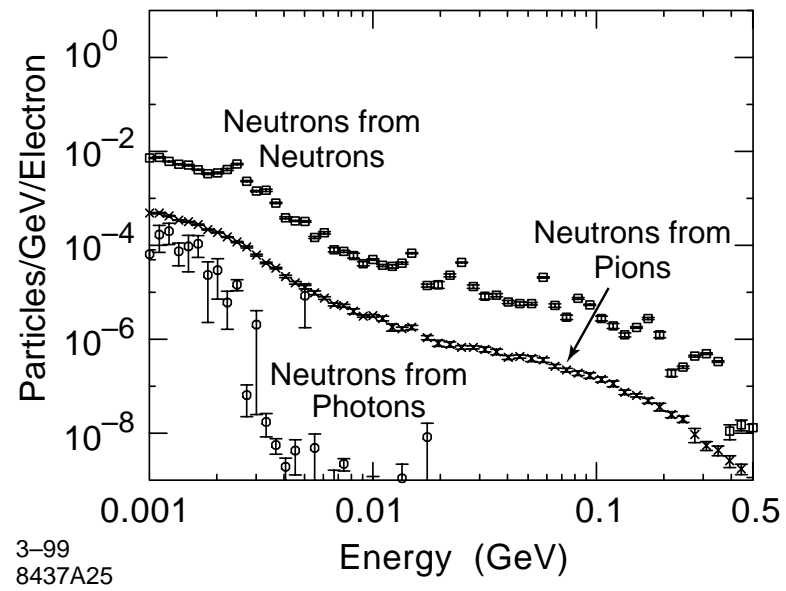

Figure 16. The sources of the neutrons created in the lead shielding (from TOTAL).

\section{Perfect Signal and Total Background}

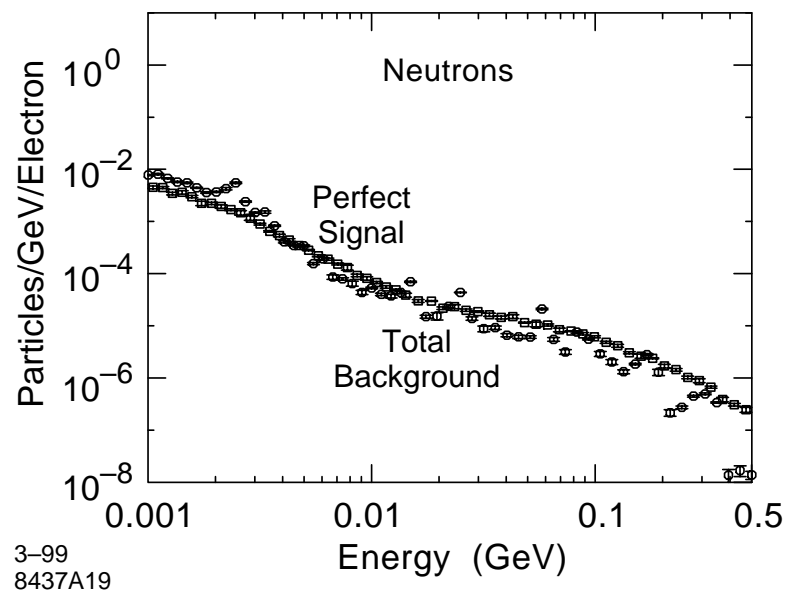

Figure 17. A comparison of the total background (from TOTAL) with the perfect signal (from SPHERES).

\section{Conclusions}

The results of this study may be summarized as follows:

(i) Through the SPHERES simulation, the photon, electron/positron, neutron, pion, and muon yields in the absence of all background have been provided (figure 9). In particular, it was found that the neutron yield drops by approximately two orders of magnitude from $2 \mathrm{MeV}$ to $100 \mathrm{MeV}$.

(ii) From the ROOM simulation, it has been found that the background caused by the scattering from the room is large, but the shape is similar to that of the signal. Further, it has been found that the photon background is dominated by the contribution from the downstream magnet, and that both the magnet and the concrete walls largely influence the neutron background.

(iii) It is apparent from the FRONT simulation that the ratio of signal to background created by the front surface of the lead shielding increases as energy increases, starting around a factor of two for energies under $5 \mathrm{MeV}$ and increasing to nearly an order of magnitude for higher energies. Further, from the BACK simulation, it has been shown that the lead shielding surrounding the detector decreases the background due to scattering from the room by around a factor of two. The combination of these two results shows that the lead shielding had an overall positive effect.

(iv) The TOTAL simulation has shown that the neutrons created in the shielding surrounding the detector are mainly the results of $(\mathrm{n}, \mathrm{xn})$ reactions and that the number of neutrons produced by photons hitting the shielding drops off dramatically at energies above $5 \mathrm{MeV}$.

(v) Finally, the TOTAL simulation shows that the ratio of the measured signal to the total background is approximately $2: 1$.

\section{References}

1. A. Fasso, A. Ferrari, J. Ranft, P.R. Sala, "FLUKA: present status and future developments", Proc. IV Int. Conf. on Calorimetry in High Energy Physics, La Biodola, Italy, 21-26 Sept. 1993, Ed. A. Menzione and A. Scribano, World Scientific, p. 493.

2. R. Mitchell, "T423 FLUKA Simulations", RP Note 99-05, March 20, 1999. 